\title{
Quantitative Susceptibility Mapping in Cerebral Cavernous Malformations: Clinical Correlations
}

\author{
(D). Tan, (D). Zhang, (D) A.G. Mikati, DR. Girard, DO. Khanna, (D) M.D. Fam, (D). Liu, (D). Wang, DR.R. Edelman, (D). Christoforidis, \\ and (DI.A. Awad \\ O* $\equiv$
}

\begin{abstract}
BACKGROUND AND PURPOSE: Quantitative susceptibility mapping has been shown to assess iron content in cerebral cavernous malformations. In this study, our aim was to correlate lesional iron deposition assessed by quantitative susceptibility mapping with clinical and disease features in patients with cerebral cavernous malformations.
\end{abstract}

MATERIALS AND METHODS: Patients underwent routine clinical scans in addition to quantitative susceptibility mapping on 3 T systems. Data from 105 patients met the inclusion criteria. Cerebral cavernous malformation lesions identified on susceptibility maps were crossverified by T2-weighted images and differentiated on the basis of prior overt hemorrhage. Mean susceptibility per cerebral cavernous malformation lesion $\left(\bar{\chi}_{\text {lesion }}\right)$ was measured to correlate with lesion volume, age at scanning, and hemorrhagic history. Temporal rates of change in $\bar{\chi}_{\text {lesion }}$ were evaluated in 33 patients.

RESULTS: Average $\bar{\chi}_{\text {lesion }}$ per patient was positively correlated with patient age at scanning $(P<.05,4.1 \%$ change with each decade of life). Cerebral cavernous malformation lesions with prior overt hemorrhages exhibited higher $\bar{\chi}_{\text {lesion }}$ than those without $(P<.05)$. Changes in $\bar{\chi}_{\text {lesion }}$ during 3- to 15-month follow-up were small in patients without new hemorrhage between the 2 scans (bias $=-0.0003 ; 95 \% \mathrm{Cl}$, $-0.06-0.06)$.

CONCLUSIONS: The study revealed a positive correlation between mean quantitative susceptibility mapping signal and patient age in cerebral cavernous malformation lesions, higher mean quantitative susceptibility mapping signal in hemorrhagic lesions, and minimum longitudinal quantitative susceptibility mapping signal change in clinically stable lesions. Quantitative susceptibility mapping has the potential to be a novel imaging biomarker supplementing conventional imaging in cerebral cavernous malformations. The clinical significance of such measures merits further study.

ABBREVIATIONS: $C C M=$ cerebral cavernous malformation; $Q S M=$ quantitative susceptibility mapping; $\bar{\chi}_{\text {lesion }}=$ mean susceptibility per cerebral cavernous malformation lesion; $\bar{\chi}_{\text {patient }}=$ lesional mean susceptibility per patient

C erebral cavernous malformation (CCM) is a common hemorrhagic vascular anomaly of the human brain, affecting $>1$ million Americans. ${ }^{1}$ Patients with CCMs are subject to $1 \%-5 \%$ annual risk and an estimated $\geq 30 \%$ lifetime risk of hemorrhage, epilepsy, seizure, and other neurologic sequelae. ${ }^{2-4}$ The clinical consequences of CCM remain unpredictable, and currently there

Received August 3, 2015; accepted after revision January 4, 2016.

From the Department of Surgery (Neurosurgery) (H.T., L.Z., A.G.M., R.G., O.K., M.D.F., I.A.A.), University of Chicago Medicine and Biological Sciences, University of Chicago, Chicago, Illinois; MedlmageMetric (T.L.), New York, New York; Department of Radiology (Y.W.), Weill Cornell Medical College, New York, New York; Department of Biomedical Engineering (Y.W.), Cornell University, Ithaca, New York; Department of Radiology (R.R.E.), NorthShore University HealthSystem, Evanston, Illinois; Department of Radiology (R.R.E.), Feinberg School of Medicine, Northwestern University, Chicago, Illinois; and Department of Radiology (G.C.), Pritzker School of Medicine, University of Chicago, Chicago, Illinois.

This project was funded, in part, by the Collaborative and Translational Studies Award through the Institute of Translational Medicine at the University of Chicago (UL1 TR000430) and the Bill and Judy Davis Research Fund in Neurovascular Research. is no known treatment to alter the course of this disease besides surgery.

Iron deposition related to hemorrhage within CCM lesions is a recognized hallmark of disease activity. ${ }^{5-7}$ Recently, therapeutic changes in lesion burden have been found with experimental drugs in animal models, in which a major phenotypic effect of the therapy was a decrease in iron deposition in lesions treated by immunohistochemistry. ${ }^{6}$ Lesional iron was therefore hypothesized to be a marker for disease progression and a relevant therapeutic target. It would be essential to determine whether the dis-

Please address correspondence to Huan Tan, PhD, Department of Surgery, Section of Neurosurgery, SBRI J328, 5841 South Maryland Ave, MC 3026, Chicago, IL 60637; e-mail: htan1@surgery.bsd.uchicago.edu

\footnotetext{
- Indicates open access to non-subscribers at www.ajnr.org

$\equiv$ Indicates article with supplemental on-line appendix.

Indicates article with supplemental on-line photo.

http://dx.doi.org/10.3174/ajnr.A4724
} 
ease severity and beneficial effect on iron can also be quantified by a method suitable for clinical studies. The current evaluation of CCM disease burden in humans primarily relies on lesion count and size with MR imaging, in great part due to the imaging of susceptibility effects caused by the iron-rich blood breakdown by-products. Modern MR imaging techniques, such as susceptibility-weighted imaging, ${ }^{8}$ can offer accurate lesion counts ${ }^{9}$; however, they lack the ability to quantify lesional iron deposition, particularly in larger clinically concerning lesions. The continuing investigation of experimental CCM therapies is in need of a quantitative technique for a more accurate and sensitive assessment of outcomes and a potential biomarker of novel therapies.

Quantitative susceptibility mapping (QSM) is a noninvasive MR imaging technique that has the potential to estimate lesional iron content by quantifying the magnetic susceptibility of local tissues. ${ }^{10,11}$ The initial feasibility of QSM in CCM was previously demonstrated in a small cohort of patients. ${ }^{12}$ Most important, QSM as a means to quantify lesional iron in CCM was validated by mass spectroscopy by using excised human lesion specimens. ${ }^{12}$ However, to our knowledge, iron burden and its evolution in relation to disease progression have not been established. Novel discoveries relating iron content and the clinical features of the disease and changes in iron accumulation with time will offer valuable information in understanding the disease pathophysiology and assisting in the development of potential interventions.

In this study, we answer those questions by applying QSM to a large cohort of patients with CCMs as a part of the routine clinical examination. We tested the following hypotheses: 1) The average CCM susceptibility per patient is correlated with age, 2) lesions with previous hemorrhagic events have higher susceptibility, and 3 ) changes in lesional susceptibility $\left(\bar{\chi}_{\text {lesion }}\right)$ in a short time $(<2$ years) are insignificant in patients who were clinically stable (ie, asymptomatic, no bleeds); other changes in QSM with time may reflect new bleeding or recovery from hemorrhage. The goal of this study was to extend from the initial feasibility ${ }^{12}$ to explore QSM for a clinical measure of the disease severity and correlate lesion-specific behaviors during disease progression.

\section{MATERIALS AND METHODS Study Design}

Clinical features of the CCM disease and prior lesion behaviors were correlated with QSM-derived iron measurements. A subcohort of patients underwent serial QSM measurements, and longitudinal assessment of QSM was correlated with clinical lesion behavior in these cases. In addition, QSM was spatially correlated to the T1- and T2-weighted imaging characteristics of lesions based on blood breakdown products. The iron sources that contribute to the QSM signal are complex. In the context of the preliminary evidence ${ }^{12}$ that demonstrated that the mean QSM signal was directly proportional to the actual iron concentration in CCM lesions by mass spectroscopy, the terms "mean susceptibility" and "iron concentration" will be used interchangeably in this article.

\section{Patients}

This study enrolled 105 consecutive patients (mean age, 39 years; range, 3-76 years) scheduled for routine clinical evaluation and
Clinical information of the included patients

\begin{tabular}{lc}
\hline \multicolumn{1}{c}{ Information } & \\
\hline No. of patients included in analysis & 95 \\
Mean age at first scan/range (yr) & $40 / 3-76$ \\
Male/female & $34: 61$ \\
No. of sporadic/familial CCMs & $39 / 56$ \\
\hline
\end{tabular}

MR imaging for CCM disease from February 2012 to December 2014. Inclusion criteria for enrollment were the presence of known CCM disease and the absence of other unrelated neurologic pathology. The imaging study took place at the University of Chicago and NorthShore University HealthSystem. Both institutional review boards approved the study, and written informed consent was obtained from each patient.

A total of 175 scans including follow-up imaging were obtained in 105 patients. Thirty-seven scans (21\%) were excluded from the final analysis due to excessive imaging artifacts because of motion and/or medical implants and the absence of lesions (eg, the scan occurred after the lesion was resected). As a result, the final analysis included 138 scans from 95 patients (96 scans in 69 patients at University of Chicago and 42 scans in 26 patients at NorthShore University HealthSystem). Among those 95 patients, 33 (22 at University of Chicago and 11 at NorthShore University HealthSystem) underwent at least 1 follow-up scan. The time between the repeat scans ranged from 91 to 455 days, with an average of 359 days.

For cross-sectional analyses, the first QSM of those who had multiple scans was used. A clinical summary of the included patients is shown in the Table.

\section{Imaging Environment and Data Acquisition}

Imaging was performed on 3T MR imaging systems. Sixty-nine patients were imaged at the University of Chicago (Achieva; Philips Healthcare, Best, the Netherlands) with an 8-channel phased array head coil, while 26 patients were imaged at NorthShore University HealthSystem (Magnetom Verio; Siemens, Erlangen, Germany) with a 12-channel phased array head coil (inter-scanner calibration was performed with satisfactory result, see On-line Appendix). Follow-up scans for patients were obtained on the same units as the ones used at their initial visits.

A 3D, T2*-weighted, multiecho, spoiled gradient-echo sequence was used for QSM data acquisition with the following common parameters: 8 TEs with uniform spacing, flip angle of $15^{\circ}$, and a parallel acceleration of a factor 2 . The following parameters are system-specific: Philips Achieva: TE, 5.6-51 ms; FOV, $224 \mathrm{~mm}$; acquisition matrix, $224 \times 224$; slab-encoding thickness, $1 \mathrm{~mm}$; TR, $66 \mathrm{~ms}$; Siemens Verio: TE, 3.6-45 ms; FOV, 240 mm; acquisition matrix, $256 \times 256$; slab-encoding thickness, $1.5 \mathrm{~mm}$; TR, $55 \mathrm{~ms}$.

\section{Data Reconstruction}

QSM images were reconstructed by using a morphology-enabled dipole inversion algorithm, ${ }^{10,13}$ which generates the local susceptibility distribution by inverting the estimated tissue field map with prior information from the magnitude images. The tissue field map was obtained by removing the background field induced by large susceptibility sources (ie, air/tissue in- 
terface) from the field map derived from the gradient-echo phase images. ${ }^{14}$ Image reconstruction was performed locally on a dedicated workstation (Intel Core i7 $2.7 \mathrm{GHz}, 16 \mathrm{~GB}$ RAM; Intel, Santa Clara, California) with an average processing time of 8 minutes.

\section{Data Analysis}

Extraction of Clinical Parameters. The electronic medical records of the patients were reviewed by 1 experienced clinician (with $>20$ years of experience in the care of CCM), and deidentified clinical information was collected and stored in a data base before initiating the correlations. Hemorrhage associated with CCM was defined according to the criteria of AlShahi Salman et al. ${ }^{15}$ Briefly, hemorrhage due to CCM requires both acute or subacute onset of symptoms accompanied by imaging evidence of acute or subacute lesional or extralesional hemorrhage. Cases were classified as familial or sporadic on the basis of lesion count, family history, and/or identified mutations by using genetic screening. ${ }^{9,16}$

Inclusion Criteria for Lesion Analysis. Lesions were selected from the 138 scans with acceptable image quality. Because sporadic cases often only contain a solitary lesion, all lesions in sporadic cases were included. In familial cases, the number of lesions can be innumerable with many small punctate lesions. Hence, we only included lesions with a maximum cross-diameter of $\geq 5$ $\mathrm{mm}^{17}$ on the corresponding $\mathrm{T} 2$-weighted images that were of higher clinical significance. A total of 407 lesions (64 from 64 scans in 39 patients with sporadic CCM and 343 from 74 scans in 56 patients with familial CCM) were included in the final analysis.

Lesion Susceptibility and Volume Measurements. CCM lesions appeared hyperintense on the QSM maps and were cross-verified with SWI and T2-weighted images. Lesion segmentation was performed by using ImageJ software (National Institute of Health, Bethesda, Maryland) by experienced scientists and physicians with high intra- and interobserver consistency demonstrated previously. ${ }^{18}$ The final ROI defined for each lesion was 3D by aggregating 2D ROI cross-multiple sections. Mean susceptibility $(\bar{\chi}=$ $\Sigma \chi_{\mathrm{ROI}} / N$, where $N$ is the number of pixels within the 3D ROI) was then calculated per lesion $\left(\bar{\chi}_{\text {lesion }}\right)$ and averaged across all lesions per patient $\left(\bar{\chi}_{\text {patient }}\right)$. Lesion volume was computed as the product of the total number of pixels in the 3D ROI and the voxel size.

Correlation Analyses. We performed correlation analysis between $\bar{\chi}_{\text {lesion }}$ (proportional to lesional iron concentration ${ }^{12}$ ) and a set of clinical parameters consisting of patients' basic characteristics and the CCM disease features. Specific parameters from basic characteristics included patient sex and age at scanning, which provide an estimation of the duration of lesion presence. Often lesion genesis can occur before the lesion becomes symptomatic; we assumed that older patients were more likely to have had the lesion for a longer duration. Parameters from the disease feature included lesional volume, sporadic versus familial, and hemorrhagic history. In patients with follow-up scans, we assessed changes with time in $\bar{\chi}_{\text {lesion }}$ in the same lesions.

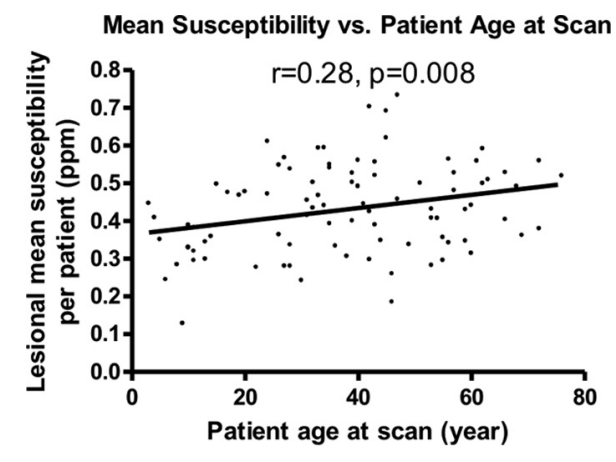

FIG 1. Lesional mean susceptibility per patient (parts per million) is positively correlated with patient age at scanning.

Spatial Correlation Based on Conventional MR Imaging Features. Spatial correlation of imaging features between QSM and conventional MR imaging sequences was conducted in 20 randomly selected lesions in our cohort. Each lesion was spatially segmented by an experienced neuroradiologist (with $>20$ years of experience) on the basis of the signal intensity on the conventional T1- and T2-weighted images as different blood breakdown products. ${ }^{19}$ We identified 4 types of blood by-products: deoxygenated hemoglobin, intra-/extracellular methemoglobin, and hemosiderin. A category of "unknown" was used to describe all other depositions within the lesion. The susceptibility of each blood by-product was empirically determined as high $(>1.4$ ppm), medium (between 0.6 and $1.4 \mathrm{ppm}$ ), or low $(<0.6 \mathrm{ppm})$. The distribution of susceptibility measurements across different blood types was analyzed.

\section{Statistical Analysis}

The Pearson correlation and Student $t$ test were applied to explore the correlations and comparisons made between $\bar{\chi}_{\text {lesion }}$ and continuous or dichotomous clinical factors, respectively. The BlandAltman plot was used to assess the changes in patients with repeat scans. Receiver operating characteristic analysis was applied to express the diagnostic accuracy of QSM identifying lesions with prior hemorrhages in cases with familial CCM. Statistical analyses were performed by using GraphPad Prism 4 software (GraphPad Software, San Diego, California), and all reported $P$ values were 2 -sided and were considered statistically significant at $P<.05$.

\section{RESULTS}

\section{Correlations with Patients' Basic Characteristics}

We found a linear, positive correlation between patient age at scanning and the $\bar{\chi}_{\text {patient }}(P<.05$, Fig 1$)$, suggesting that older lesions have a higher iron concentration. The average change in $\bar{\chi}_{\text {patient }}$ was small (estimated to be $4.8 \%$ per decade of life); this finding may imply a slow, progressive iron deposition. $\bar{\chi}_{\text {patient }}$ was not significantly different between sexes. Furthermore, there was no correlation between $\bar{\chi}_{\text {patient }}$ in symptomatic patients and years since initial symptom onset.

\section{Correlations with Clinical Features of the Disease}

Sporadic and Familial CCM and Lesion Volume. We did not find a significant difference in $\bar{\chi}_{\text {patient }}$ between patients with sporadic and familial CCM. No correlation was found between $\bar{\chi}_{\text {lesion }}$ and 

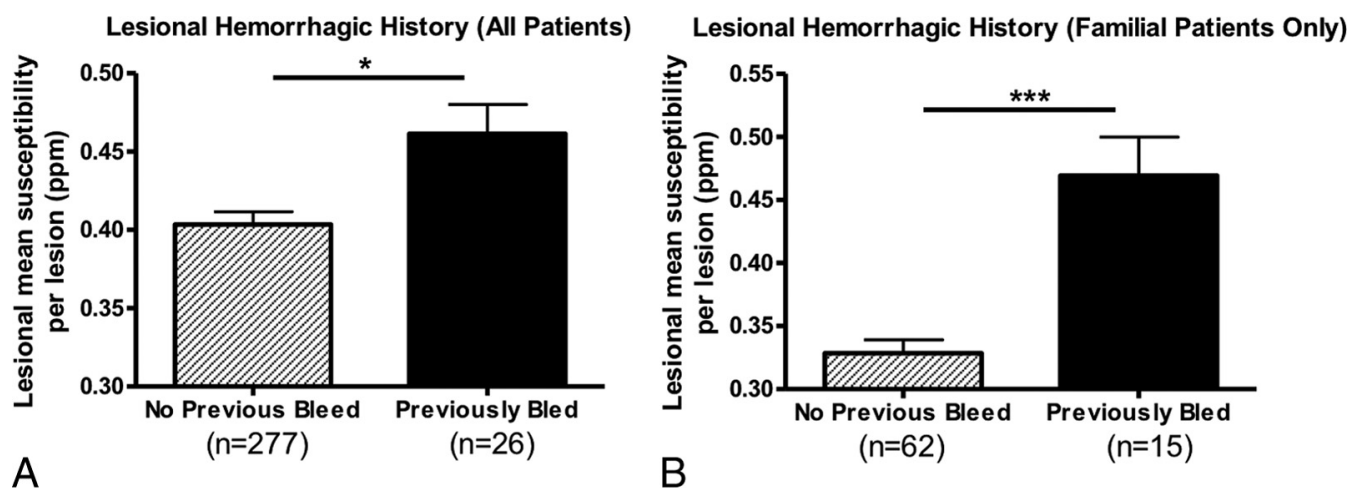

ROC Curve (Familial Patients Only) AUC $=0.83, p<0.001$
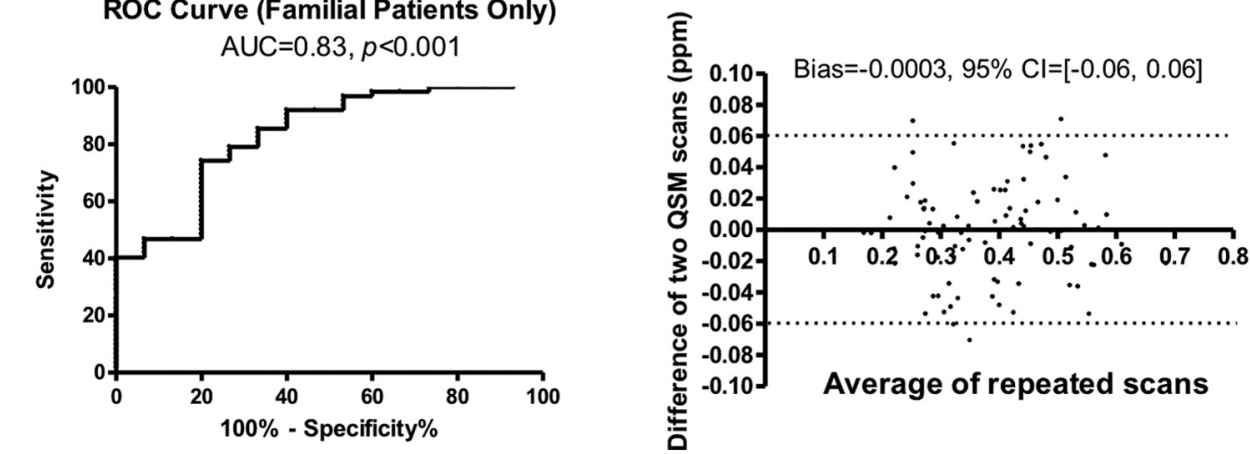

C

FIG 2. A, Mean susceptibility comparison between lesions with and without prior hemorrhages in all patients. B, Mean susceptibility comparison between lesions with and without prior hemorrhages only in patients with familial CCM. C, Receiver operating characteristics analysis indicates that QSM is a good differentiator of lesions with and without prior hemorrhage in cases with familial CCM. D, A Bland-Altman plot of the repeat QSM measurements. Changes in susceptibility were small in clinically stable patients within a short follow-up.

lesion volume, indicating that lesional iron concentration is independent of lesion volume.

Hemorrhagic and Nonhemorrhagic Lesions. CCM lesions in patients with both sporadic and familial CCMs with previous overt hemorrhages were found to have significantly higher iron concentrations. $\bar{\chi}_{\text {lesion }}$ in 26 lesions that previously bled at least once was significantly higher than that in lesions $(n=277)$ that had no history of prior CCM hemorrhages $(P<.05$, Fig $2 A)$. The $95 \%$ confidence interval for the difference between patients with and without overt hemorrhage was $0.39-0.50$. This result suggests that CCM lesions with prior symptomatic bleeds have more iron or iron-containing products.

The difference in $\bar{\chi}_{\text {lesion }}$ between hemorrhagic and nonhemorrhagic cases was more prominent in patients with familial CCM $(P<.01$, Fig 2B). Considering lesions with prior hemorrhagic events as the true-positive group and the remaining lesions as the true-negative group in our data, we performed receiver operating characteristic analysis as a measure of the overall discriminative performance of QSM - that is, the overall ability to identify lesions with prior bleeds ahead of those without. The area under curve was 0.83 , suggesting that QSM had a good accuracy in identifying prior hemorrhage of a lesion (Fig 2C).

When we controlled for lesion volume, the difference in $\bar{\chi}_{\text {lesion }}$ was not significant between hemorrhagic and nonhemorrhagic lesions. This finding suggests that the effect of prior hemorrhages on lesional iron concentration is mediated, at least in part, by lesion volume.

\section{Longitudinal Changes in Lesional Susceptibility}

Changes in $\bar{\chi}_{\text {lesion }}$ were small among 33 patients in the short term (mean, $300 \pm 125$ days; median, 360 days). The bias for changes in mean susceptibility was -0.0003 ( $95 \%$ confidence interval, -0.06-0.06), shown in the Bland-Altman plot (Fig 2D). Among the 33 patients, lesions from 20 patients were clinically stable without historical symptoms (bias $=-0.003$; 95\% CI, $-0.064-$ 0.058); 5 patients had a CCM-related hemorrhage within 6 months before the first QSM (bias $=-0.015$; 95\% CI, $-0.033-$ $0.064)$; and 8 patients had a CCM-related hemorrhage $>6$ months before the first QSM (bias $=0.23$; 95\% CI, $-0.044-$ 0.075). None of the patients experienced a hemorrhage between their first and second QSM scans. In the same cohort, no significant correlation was found between changes in $\bar{\chi}_{\text {lesion }}$ and changes in lesion volume, indicating that changes in lesional iron concentration can occur independent of changes in lesion volume.

\section{Impact of an Operation and Recent Hemorrhage on QSM}

In 1 patient with sporadic CCM (a 41-year-old woman), the lesion located in the right frontal lobe was removed surgically before the second QSM scan. The success of the operation was reflected on the QSM image, where only residual iron deposition was seen at the edge of the old lesion (Fig $3 A$ ).

One patient with sporadic CCM (a 28-year-old woman), in whom the CCM lesion bled 3 months before her first QSM scan in September, had a rebleed in the same lesion in October. A second QSM scan was obtained in December, and an increase in both $\bar{\chi}_{\text {lesion }}$ 

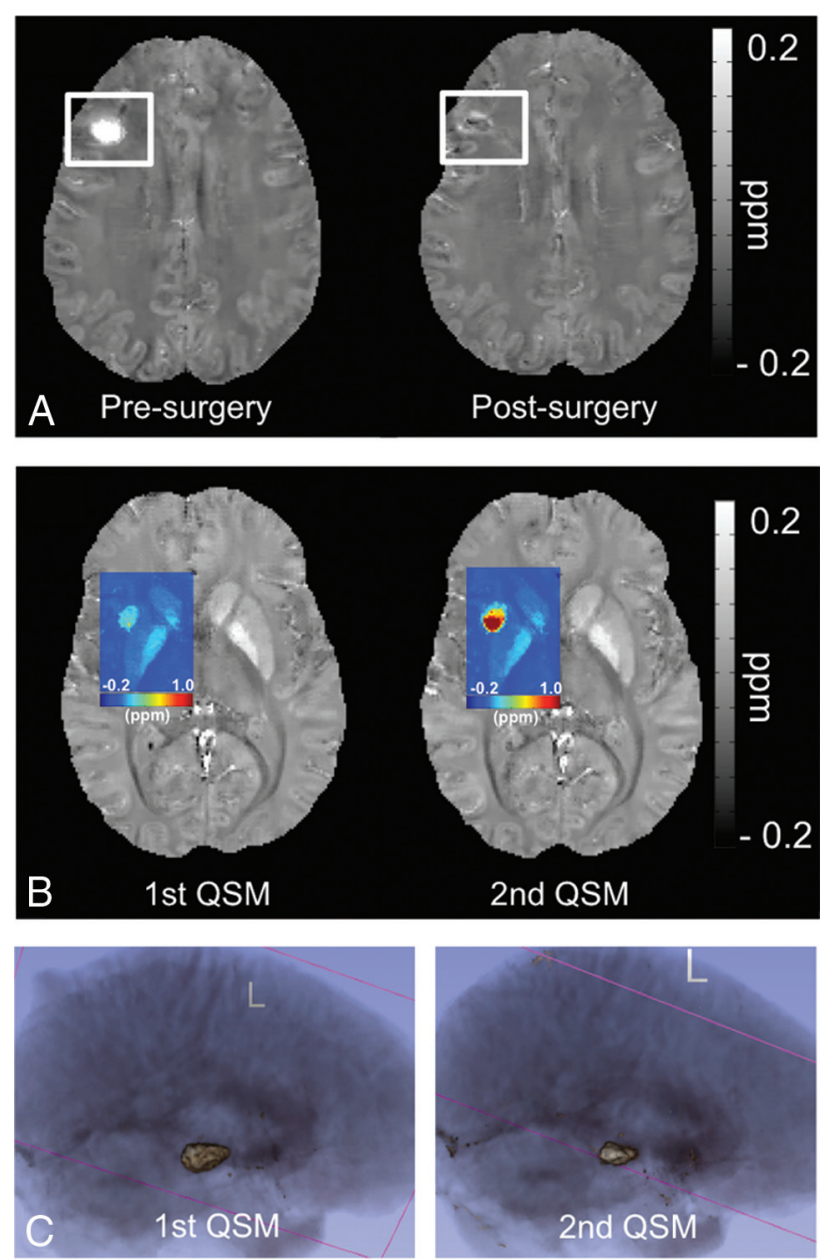

FIG 3. Each subfigure exhibits a separate CCM case. A, QSM map depicts the appearance of a CCM lesion before and after the operation. $B$, A colorized QSM map depicting a big increase in iron deposition in the same CCM lesion in a short time, after 2 recent overt hemorrhages. C, 3D rendering of a CCM lesion in repeat scans after a known hemorrhage, depicting the shrinkage in overall lesion volume during the short-term recovery period in a patient following a hemorrhage, but with increased mean susceptibility.

(from 0.1751 to $0.4659 \mathrm{ppm}$ ) and lesion volume (from 519 to 932 $\mathrm{mm}^{3}$ ) was observed (Fig $3 B$ ). We suspect that the increase in lesional iron deposition was related to the 2 overt hemorrhages in such a short timeframe. One other patient with familiar CCM (a 2-year-old boy) had a hemorrhage 11 months after the first QSM scan with a second QSM follow-up 2 months later. However, no significant change was observed in the lesion that bled ( $\bar{\chi}_{\text {lesion, } 1 \text { st QSM }}=0.4966 \mathrm{ppm}$, first lesion volume $=3960 \mathrm{~mm}^{3} ; \bar{\chi}_{\text {lesion, } 2 \mathrm{nd} \mathrm{QSM}}=0.4765 \mathrm{ppm}$, second lesion volume $=3919 \mathrm{~mm}^{3}$ ).

One other case worth noting was a 59-year-old patient with sporadic CCM who underwent her first QSM scan 14 days after the lesion bled, and a second QSM scan was performed 35 days after that. Although both the lesion volume and the total susceptibility decreased from 2165 to $1242 \mathrm{~mm}^{3}$ and from 1130 to 723 ppm $\times \mathrm{mm}^{3}$, respectively (Fig $3 C$ ), $\bar{\chi}_{\text {lesion }}$ increased slightly from 0.4298 to $0.4791 \mathrm{ppm}$. This change suggests that the human body does not effectively remove the iron products associated with hemorrhage during the natural recovery progress. Furthermore, this case illustrates that changes in lesion volume do not necessarily reflect a concomitant change in lesional iron concentration.

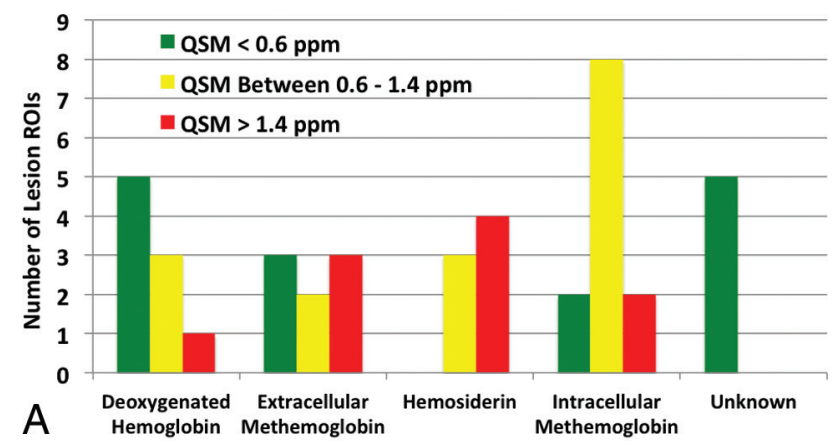

QSM
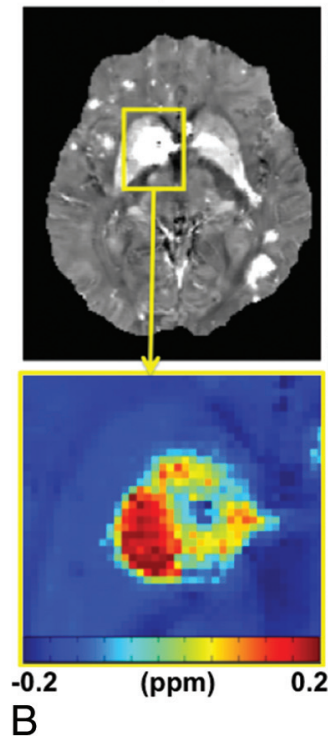

Conventional MRI

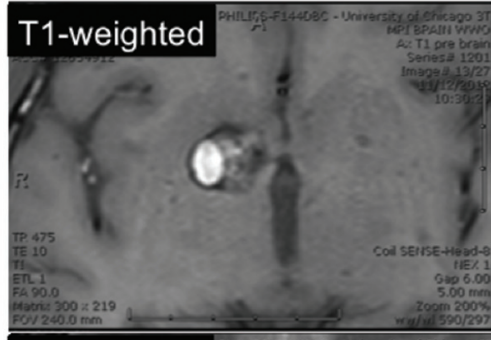

T2-weighted

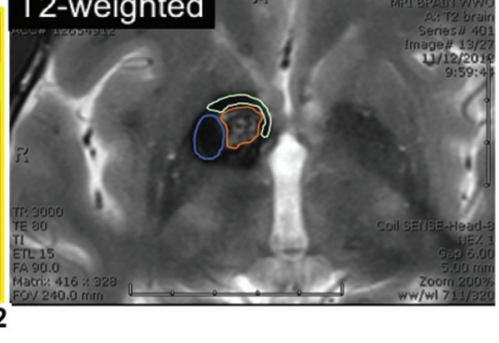

FIG 4. Spatial comparison results between QSM and conventional MR imaging. A, Susceptibility distribution of different blood breakdown by-products within CCM lesions. B, Illustration of blood breakdown by-product identification and correlation with the corresponding QSM measurements. ROls for different blood by-products are shown in the T2-weighted image with color (blue, intracellular methemoglobin; brown, intracellular methemoglobin and hemosiderin; cyan, hemosiderin).

Overall, of the 3 patients with follow-up QSM after recent hemorrhages in a longitudinal study, 2 had an interval increase in lesional iron concentrations without an increase in lesion volume and a third had nearly 2.5 -fold increase in lesional iron concentration, with only an $80 \%$ increase in lesion volume. This finding shows that increased iron concentration in CCM lesions can occur after hemorrhage, independent of lesion volume.

\section{Spatial Correlation with Conventional MRI}

All CCM lesions revealed hypointense signal compared with normal brain parenchyma on QSM. A total of 41 ROIs were identified from 20 randomly selected CCM lesions, consisting of 9 deoxygenated hemoglobin, 8 extracellular methemoglobin, 12 intracellular methemoglobin, 7 hemosiderin, and 5 unknown regions. The signal intensities for deoxygenated hemoglobin, extracellular methemoglobin, and intracellular methemoglobin spanned the full spectrum (low to high) on the QSM images, whereas hemosiderin exhibited medium and high intensities, and the unidentified regions exhibited low intensities (Fig 4A). In particular, hemosiderin demonstrated the most homogeneous signal distri- 
bution on QSM, while the other blood by-product regions exhibited both homogeneous and heterogeneous signal distributions. An example of the spatial correlation between QSM and the conventional T1 and T2 images is shown in Fig $4 B$.

\section{DISCUSSION}

Our previous investigation ${ }^{12}$ demonstrated the proof of concept that QSM can quantify lesional iron deposition in CCM. In the current study, we aimed to evaluate the relationship between iron burden by using QSM and the clinical features of the CCM disease in a larger clinical population.

Because the total iron content measured by QSM in CCM lesions is inherently related to the lesion size (ie, larger lesions contain more iron deposition ${ }^{12}$ ), we sought to assess lesional mean susceptibility, a measurement proportional to the lesional iron concentration, as the metric to assess lesional iron burden. The results have confirmed the hypothesis that lesions in older patients had higher iron burden. We used age at scanning as an estimate for the duration of lesion presence because lesion genesis is unknowable in most cases. Other studies have analyzed bleed risk and other natural history features on the basis of similar years of exposure since birth. ${ }^{3,20}$ Aging itself may be a factor in this observation, given the increasing evidence to show that older brains are associated with increased iron. ${ }^{21}$ This observation supports the conservation of mass hypothesis, with QSM signal reflecting lesional permeability as demonstrated in a previous study, ${ }^{18}$ now also reflected by iron accumulation with time.

No correlation was found between lesional iron concentration and lesion volume. This finding confirmed our initial observation in the prior feasibility study. ${ }^{12}$ Changes in lesional volume and iron concentration may be 2 independent indicators of different aspects of disease activities, which will be further examined in a future prospective longitudinal investigation.

We found that lesions with prior overt hemorrhages were shown to have a higher iron concentration than those without. Even though the observation was predominately in cases of familial CCM, it still indicates that hemorrhages were related to iron depositions within CCM lesions, and it confirmed that the human body lacks an effective mechanism to clear the residual iron products from a CCM during recovery. The receiver operating characteristic analysis suggests that QSM might identify prior bleeding events in cases of familial CCM with a good sensitivity measure. This aspect of the QSM application is important, especially when a patient's medical history is unknown or when patients do not present for medical evaluation after unknowingly experiencing a hemorrhage. While the mean iron concentration in bled lesions was higher than ones without bleeds in cases with sporadic CCM, it did not reach statistical significance. This outcome may be related to the small sample size of sporadic bleeds.

Our cross-sectional data showed that the hemorrhagic effect on the lesional iron concentration was mediated, at least in part, by lesion volume in cross-sectional observations. This cohort may not have included a sufficient number of hemorrhagic cases in all volume strata. Our data from the longitudinal study in patients with repeat QSM scans after recent hemorrhage showed a manifest increase in lesional iron concentrations independent of lesional volume. To fully evaluate whether QSM offers an added measure beyond lesion size, a higher number of patients with hemorrhage with repeat scans are required.

The longitudinal results in cases with repeat QSM studies revealed little temporal change in lesional iron for up to 15 months in clinically stable lesions (Fig 2D). Lesions included in the analysis consist of those without any prior hemorrhages and those with prior hemorrhage in the chronic stage before the first QSM. In both scenarios, the susceptibility signal within the lesion remained relatively unchanged at short-term follow-up. The observation of minimum change in stable CCM lesions and increased susceptibility signal associated with overt hemorrhages may indicate that lesional iron concentration increases only in the presence of recent bleeds. This hypothesis will be subject to more rigorous testing in a larger longitudinal study.

We found that there was a large variation (estimated range, $0.4-1.9 \mathrm{ppm}$ ) in the susceptibility signal within regions of different blood breakdown products (eg, deoxygenated hemoglobin, intracellular methemoglobin, extracellular methemoglobin). Hemosiderin, however, consistently exhibited a higher mean susceptibility value $(>1.0 \mathrm{ppm})$. This could be the result of hemosiderin crystallization generating superparamagnetism. ${ }^{22}$ The heterogeneity and variation of susceptibility values in deoxygenated hemoglobin, intracellular methemoglobin, and extracellular methemoglobin suggest that there might be other contributing susceptibility sources. Overall, we believe that the spatial distribution of the lesional susceptibility does offer additional information beyond that from conventional MRI, the details of which require further investigation.

Limitations of this study include lesional iron content, which may be underestimated due to partial volume effects because other susceptibility sources such as diamagnetic myelin were not accounted for. This study was not powered to detect the effects of lesional iron content in patients recovering from or with recent bleeds, though the ongoing study is currently accumulating additional data to power future studies in relation to ongoing CCM lesion activity. The classification of blood by-products based on Bradley $^{19}$ was overly simplified. Differentiating various blood products was a difficult task, and some ROI regions contained spatially heterogeneous signal distribution on conventional MRI. Those regions were likely to contain a variety of blood iron products, and they were classified according to the predominant signal appearances. In addition, we did not specifically examine cases of CCM associated with seizures, in which QSM may reflect lesional epileptogenicity, and this will be addressed in future studies. While the data in this study were not enough to establish the clinical utility of QSM, we postulate that QSM may still be used as a potential imaging biomarker to supplement information from conventional imaging and to calibrate experimental therapies in future clinical trials targeted at reducing lesional iron deposition in CCM.

\section{CONCLUSIONS}

We demonstrated in the current study the following: 1) lesional mean susceptibility was positively correlated with patient age, 2) lesions with prior symptomatic bleeding have higher mean susceptibility than those without, and 3) changes in lesional susceptibility were minimal in clinically stable CCM lesions. Addition- 
ally, in a limited number of observations, there was a significant increase in lesional mean susceptibility in association with new clinical hemorrhage, motivating further the prospective longitudinal investigation already underway. The findings in this study will be hypothesis-generating for future investigations that will help guide and design human trials for potential treatments.

\section{ACKNOWLEDGMENTS}

The authors thank Eugene Dunkle and Dr Wei Li for assisting with data collection and the Center for Advanced Imaging at NorthShore University HealthSystem for providing the scanning time and equipment for the study.

Disclosures: Huan Tan—RELATED: The project was funded in part by the Collaborative and Translational Studies Award through the Institute of Translational Medicine at the University of Chicago (UL1 TR000430) and by the Bill and Judy Davis Research Fund in Neurovascular Research*; OTHER RELATIONSHIPS: Drs Tian Liu and Yi Wang have filed patents related to QSM technology. Dr Tian Liu is an employee of MedlmageMetric in New York. Tian Liu—RELATED: Grant: Medlmagemetric, ${ }^{*}$ Comments: National Institutes of Health grant 1 R43 EB015293-01; UNRELATED: Patents (planned, pending, or issued): I am listed as an inventor on patent applications related to QSM. Yi Wang-RELATED: Grant: National Institutes of Health R01NS072370*; UNRELATED: Patents (planned, pending, or issued): I am one of the inventors of QSM; Stock/Stock Options: MedlmageMetric, Comments: interested in QSM commercialization. Robert R. Edelman-UNRELATED: Grants/Grants Pending: National Institutes of Health, Siemens Healthcare; Patents (planned, pending, or issued): Noncontrast MR angiography; Royalties: Siemens Healthcare. Gregory ChristoforidisRELATED: Grant: National Institutes of Health.* Issam A. Awad-RELATED: Grant: National Institutes of Health/National Institute of Neurological Disorders and Stroke, ${ }^{*}$ Davis Research Fund in Neurovascular Surgery Research*; UNRELATED: Expert Testimony: legal expert testimony, unrelated to subject of research; Grants/ Grants Pending: National Institutes of Health/National Institute of Neurological Disorders and Stroke.* *Money paid to the institution.

\section{REFERENCES}

1. Barrow D, Krisht A, AANS Publications Committee. Cavernous malformation and hemorrhage. In: Awad IA, Barrow D, eds. Cavernous Malformations. Park Ridge: American Association of Neurological Surgeons; 1993:65-80

2. Bruner JM, Tien RD, McLendon RE. Tumors of vascular origin. In: Bigner DD, McLendon RE, Bruner JM, et al, eds. Russell and Rubinstein's Pathology of Tumors of the Nervous System. London: Arnold; 1998:239-93

3. Robinson JR, Awad IA, Little JR. Natural history of the cavernous angioma. J Neurosurg 1991;75:709-14 CrossRef Medline

4. Robinson JR Jr, Awad IA, Magdinec M, et al. Factors predisposing to clinical disability in patients with cavernous malformations of the brain. Neurosurgery 1993;32:730-35; discussion 735-36 CrossRef Medline

5. McDonald DA, Shenkar R, Shi C, et al. A novel mouse model of cerebral cavernous malformations based on the two-hit mutation hypothesis recapitulates the human disease. Hum Mol Genet 2011; 20:211-22 CrossRef Medline

6. McDonald DA, Shi C, Shenkar R, et al. Fasudil decreases lesion bur- den in a murine model of cerebral cavernous malformation disease. Stroke 2012;43:571-74 CrossRef Medline

7. Shenkar R, Venkatasubramanian PN, Zhao JC, et al. Advanced magnetic resonance imaging of cerebral cavernous malformations, part I: high-field imaging of excised human lesions. Neurosurgery 2008; 63:782-89; discussion 789 CrossRef Medline

8. Haacke EM. Susceptibility weighted imaging (SWI). Z Med Phys 2006;16:237 CrossRef Medline

9. de Champfleur NM, Langlois C, Ankenbrandt WJ, et al. Magnetic resonance imaging evaluation of cerebral cavernous malformations with susceptibility-weighted imaging. Neurosurgery 2011;68: 641-47; discussion 647-48 CrossRef Medline

10. Liu T, Liu J, de Rochefort L, et al. Morphology enabled dipole inversion (MEDI) from a single-angle acquisition: comparison with COSMOS in human brain imaging. Magn Reson Med 2011;66: 777-83 CrossRef Medline

11. Schweser F, Deistung A, Lehr BW, et al. Quantitative imaging of intrinsic magnetic tissue properties using MRI signal phase: an approach to in vivo brain iron metabolism? Neuroimage 2011;54: 2789-807 CrossRef Medline

12. Tan H, Liu T, Wu Y, et al. Evaluation of iron content in human cerebral cavernous malformation using quantitative susceptibility mapping. Invest Radiol 2014;49:498-504 CrossRef Medline

13. Liu T, Wisnieff C, Lou M, et al. Nonlinear formulation of the magnetic field to source relationship for robust quantitative susceptibility mapping. Magn Reson Med 2013;69:467-76 CrossRef Medline

14. Liu T, Khalidov I, de Rochefort L, et al. A novel background field removal method for MRI using projection onto dipole fields (PDF). NMR Biomed 2011;24:1129-36 CrossRef Medline

15. Al-Shahi Salman R, Berg MJ, Morrison L, et al; Angioma Alliance Scientific Advisory Board. Hemorrhage from cavernous malformations of the brain: definition and reporting standards-Angioma Alliance Scientific Advisory Board. Stroke 2008;39:3222-30 CrossRef Medline

16. Abdulrauf SI, Kaynar MY, Awad IA. A comparison of the clinical profile of cavernous malformations with and without associated venous malformations. Neurosurgery 1999;44:41-46; discussion 46-47 CrossRef Medline

17. Campbell PG, Jabbour P, Yadla S, et al. Emerging clinical imaging techniques for cerebral cavernous malformations: a systematic review. Neurosurg Focus 2010;29:E6 CrossRef Medline

18. Mikati AG, Tan H, Shenkar R, et al. Dynamic permeability and quantitative susceptibility: related imaging biomarkers in cerebral cavernous malformations. Stroke 2014;45:598-601 CrossRef Medline

19. Bradley WG Jr. MR appearance of hemorrhage in the brain. Radiology 1993;189:15-26 CrossRef Medline

20. Shenkar R, Shi C, Rebeiz T, et al. Exceptional aggressiveness of cerebral cavernous malformation disease associated with PDCD10 mutations. Genet Med 2015;17:188-96 CrossRef Medline

21. Zecca L, Youdim MB, Riederer P, et al. Iron, brain ageing and neurodegenerative disorders. Nat Rev Neurosci 2004;5:863-73 CrossRef Medline

22. Richter GW. Electron microscopy of hemosiderin; presence of ferritin and occurrence of crystalline lattices in hemosiderin deposits. J Biophys Biochem Cytol 1958;4:55-58 CrossRef Medline 